\title{
Vehicle Modelling and Simulation in Simulink
}

\author{
A. SZÁNTÓ1 ${ }^{1}$ S. HAJDU ${ }^{2}$ \\ 1University of Debrecen, Faculty of Engineering, andras.szanto.0503@gmail.com \\ 2University of Debrecen, Faculty of Engineering, hajdusandor@eng.unideb.hu
}

Abstract. In this paper a vehicle dynamics model is presented, which is an example that contains all the necessary aspects of making a decent vehicle model. Several examples show the use of such a model: basic vehicle dynamics phenomena can be recognized with the simulation of a detailed vehicle model. We are dealing with the connection between downforce and under/oversteer in this paper. In addition, the use of numerical simulations in the field of control systems is pointed out by an example of simulating an ABS control for the vehicle.

\section{Introduction}

Model-based design requires adequate models for simulation. The details of these models depend on the purpose of the simulation. In several cases, a simplified vehicle model - in which the vehicle is modelled with one translational freedom - is good enough for the simulation and optimization. However, there are some cases when it is necessary to model the suspension of the vehicle, and modelling only the longitudinal forces of the wheels is not enough. In this case, rigid body dynamics are applied to create the mechanism of the vehicle suspension. There are several environments in which we can create rigid body systems without creating the ODE/DAE systems on our own. In this paper, MATLAB/Simulink/Simscape Multibody is used. In the case of real time simulation demands, the linearly implicit Euler method is used [1] to solve stiff ODE/DAE systems: in the field of vehicle models, modelling the wheels usually results in stiff systems. Simscape provides tools to import complete 3D assemblies from CAD softwares as rigid body systems: to demonstrate this, a vehicle model [2] with MacPherson suspension is imported from Solidworks. Vehicle tires are usually quite complex parts: there are simplifications [3] with which additional DOF are added to the rigid body system to approximate better the tire behavior. The modelling of the contact between the tire and the ground is another task: there are quite sophisticated ways [4] to do it, but in this example, a simple approach is applied based on an official Simulink library [5]. Moreover, the semi-empirical Pacejka wheel model [6] is applied to the vehicle, which is an adequate model modelling 3 forces and 3 torques in the contact point. Because of the large number of constant parameters, it is necessary to arrange properly the whole model: for this purpose, several practises are shown in this paper [7]. The rest of the paper shows aspects of using the prepared numerical vehicle model. Simple tests are analysed to show basic vehicle dynamics phenomena as well as the simulation possibilities of control systems are also pointed out with modelling an ABS control.

In [8] the history of unmanned ground vehicles (UGVs) is presented and unmanned aerial vehicles (UAVs) are also discussed in [9]. The history of the Support Vector Machine (SVM) method and fuzzy 
logic and its use for classification and regression calculation is described in this [10] paper. Electric vehicles' battery parameter tolerances are analysed by fuzzy logic in this [11] study.

A computer program is shown [12] in which the performance parameters of electromobiles are calculated. The equation of motion of pneumatic vehicles [13] is also presented after determining the basic characteristics of the engine of a pneumatic driven mobile. Dynamic simulation of a series wound DC motor [14] can also be connected with vehicle simulations, the main object of this article.

\section{Rigid body system of the vehicle geometry}

The rigid body system that can represent the geometry and mass properties of the vehicle is modelled in the above-mentioned Simscape Multibody environment in MATLAB/Simulink. Importing the assembly [2] is a good choice but personally, it is better to build the whole mechanism from sketch. This way it is easier to define the properties of the mechanism in a MATLAB struct. CAD design can be made based on the coordinate systems of each part in the rigid body model. It is important to assign clearly a coordinate system for each part.

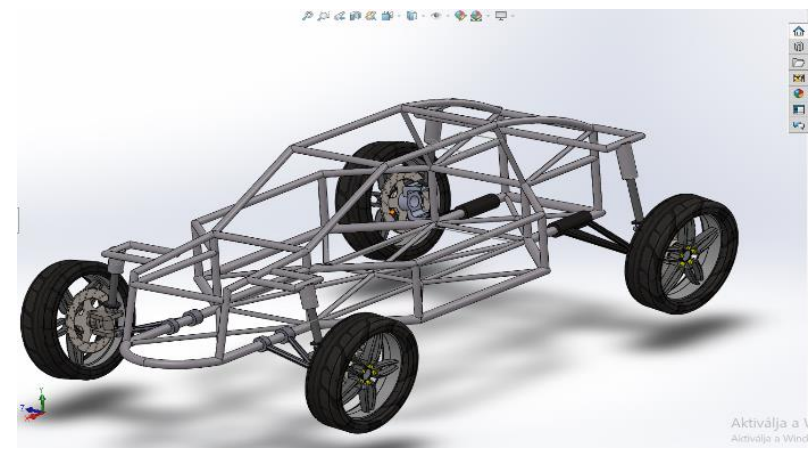

Figure 1. The imported assembly in Solidworks
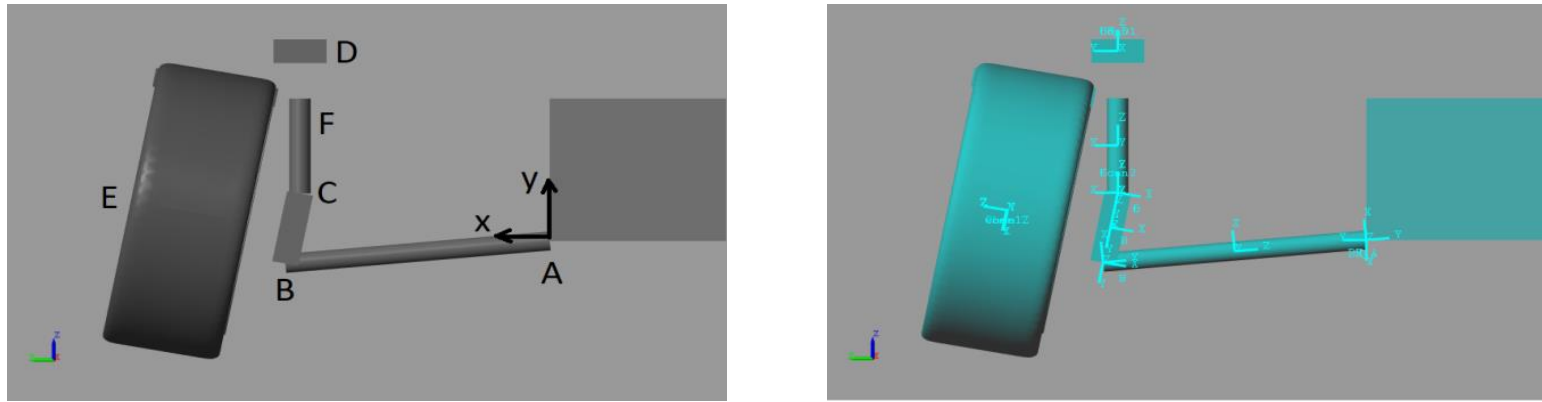

Figure 2. MacPherson suspension geometry sketch and the coordinate systems in Simscape

The tire simplification [3] in the rigid body system is modelled with an additional translational and rotational freedom between the rim and the tire.
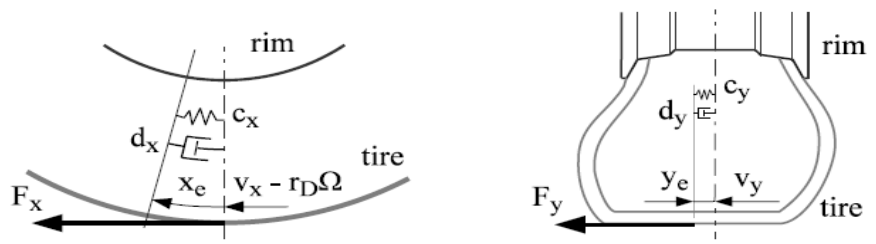

Figure 3. The additional 2 DOF (source: [3]) 


\section{Contact modelling}

A simple approach for modelling contacts is based on stiffness and damping forces. In the downloaded library [5], there are contact blocks in which contact forces can be applied between simple geometries assigned to any Simscape reference frames. For example, the intersection depth and its velocity in the case of a sphere-plane contact can be easily calculated and linear or any other force characteristics can be applied.

\section{Wheel model}

The semi-empirical Pacejka wheel model [6] is a precise wheel model because it can calculate all forces and torques reduced in the contact point. Because of the contact point reduction, the computational time is relatively fast. In this vehicle model, the normal force of the tires (Fz) is based on the contact modelling, so the other 5 forces/torques come from the wheel model. This wheel model contains 150 constant parameters per wheel, so it can be set to any real tires. These forces and torques are: Fx (longitudinal force), Fy (side/lateral force), Fz (normal force: based on the contact), Mx (overturning couple), My (rolling resistance), Mz (aligning torque).

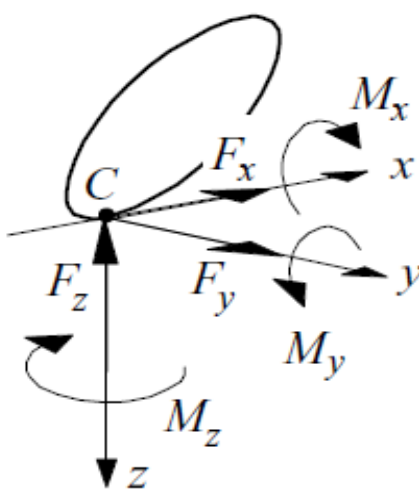

Figure 5. Wheel model directions (source: [6])

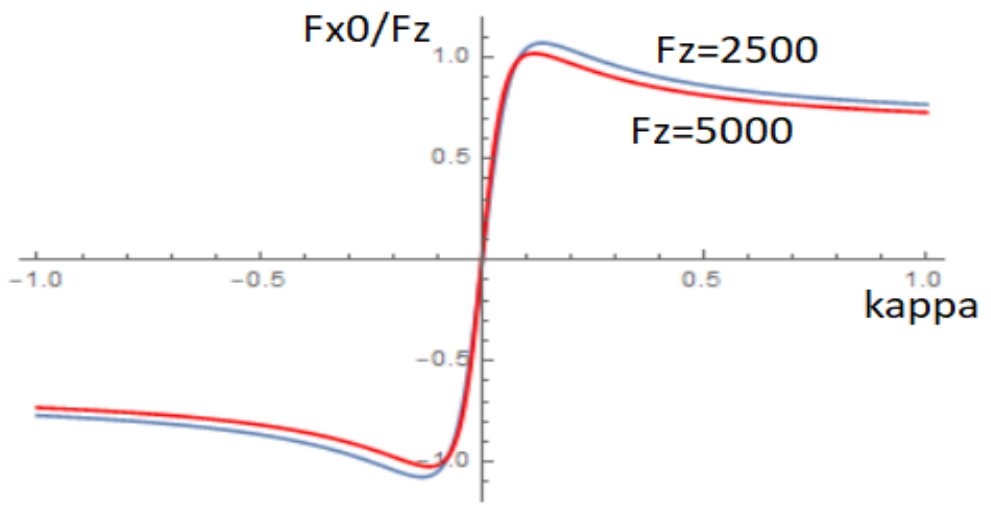

Figure 4. Pure longitudinal force ( $\mathrm{Fx} 0$ ) is not linearly proportional to the normal force $(\mathrm{Fz})$. Kappa is the longitudinal slip.

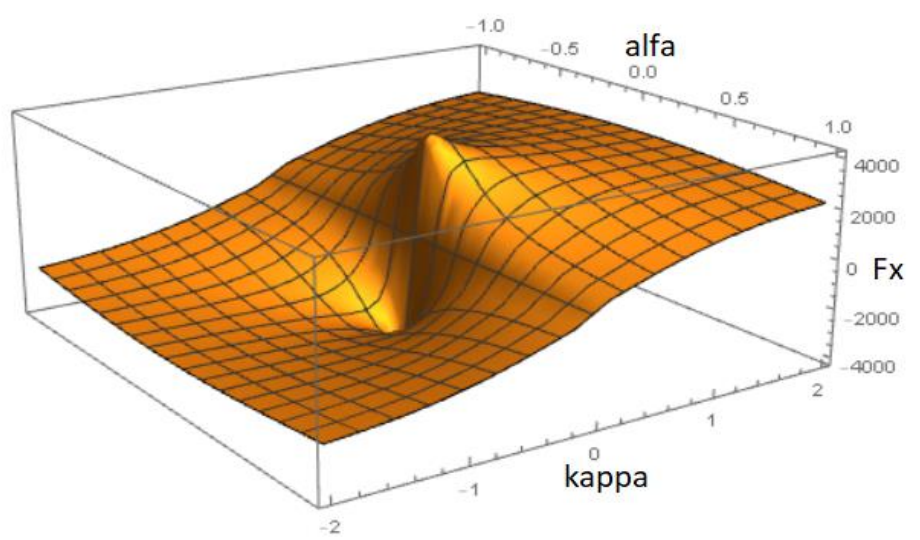

Figure 6. Longitudinal force ( $F x$ ) in combined slip scenarios (kappa is the longitudinal, and alfa is the side slip). 


\section{Summary of the vehicle model}

The whole model contains approximately 800 constant parameters. Therefore, it is important to arrange [7] properly the model in Simulink and it is good to load these constant parameters from a MATLAB script. Additional blocks and models can be added easily to a well-built and transparent model.

\begin{tabular}{|c|c|}
\hline Field - & Value \\
\hline$\square$ timeStep & $1.0000 \mathrm{e}-03$ \\
\hline HimTime & 20 \\
\hline -E simscape & $1 \times 1$ struct \\
\hline E E susp & $1 \times 1$ struct \\
\hline -E contact & $1 \times 1$ struct \\
\hline EE wheel & $1 \times 1$ struct \\
\hline
\end{tabular}

Figure 7. MATLAB struct with all constants

\section{Effect of downforce}

Understeer and oversteer are well-known phenomena in vehicle dynamics. These can be defined with real and theoretical turning radiuses. The theoretical turning centre is the point where the wheel axes intersect each other on the plane the vehicle moves on. The real turning centre is based on the velocity and acceleration vector of the vehicle. The downforce is modelled with a front and a rear force in the local z (up) direction of the vehicle body.
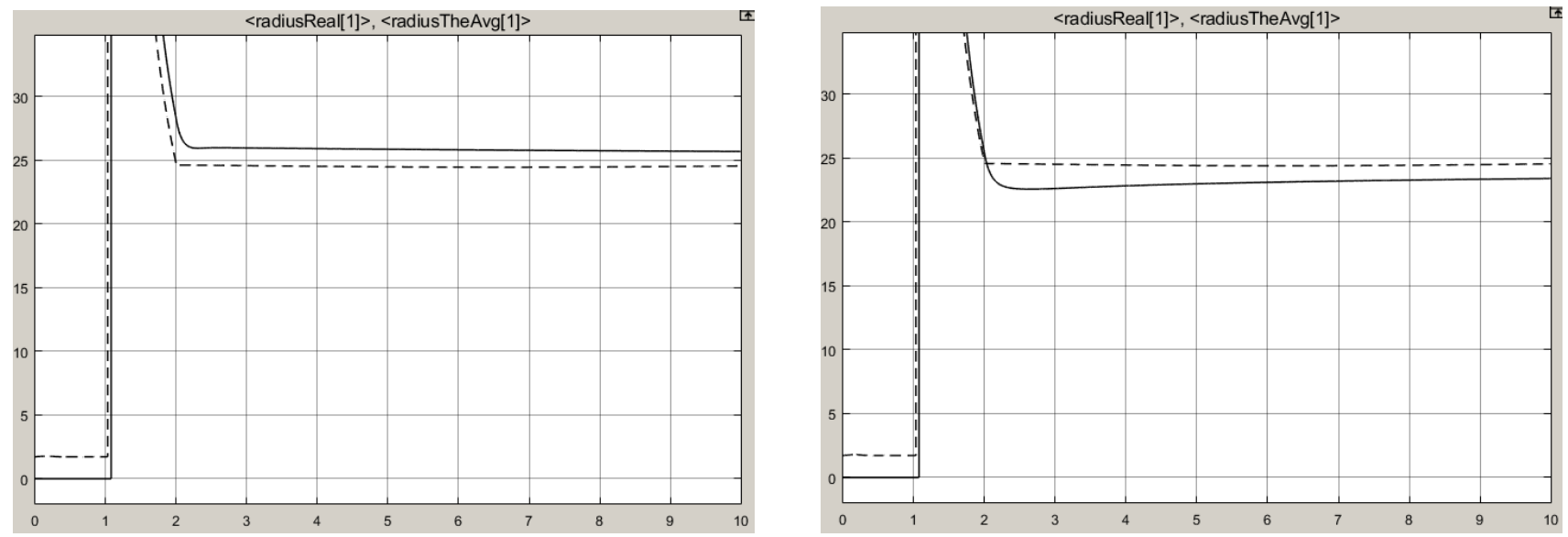

Figure 8. Real and theoretical (dotted) turning radiuses: -1000N downforce on the rear (left) and at the front (right)

\section{Modelling of an ABS control}

The created vehicle model has 12 base inputs: wheel torques, brake forces, steering angles and downforces. These inputs can be defined manually or can be resulted by another model that can use all vehicle states. This theoretical ABS model is applied with this approach. It is a theoretical model because the actuator is capable of a linear increase, decrease or hold in the value of the brake force of 
the wheel. The ABS algorithm is based on slip thresholds and controls the actuators. The slip values used in the algorithm are delayed because the actuator is not real and responses immediately.

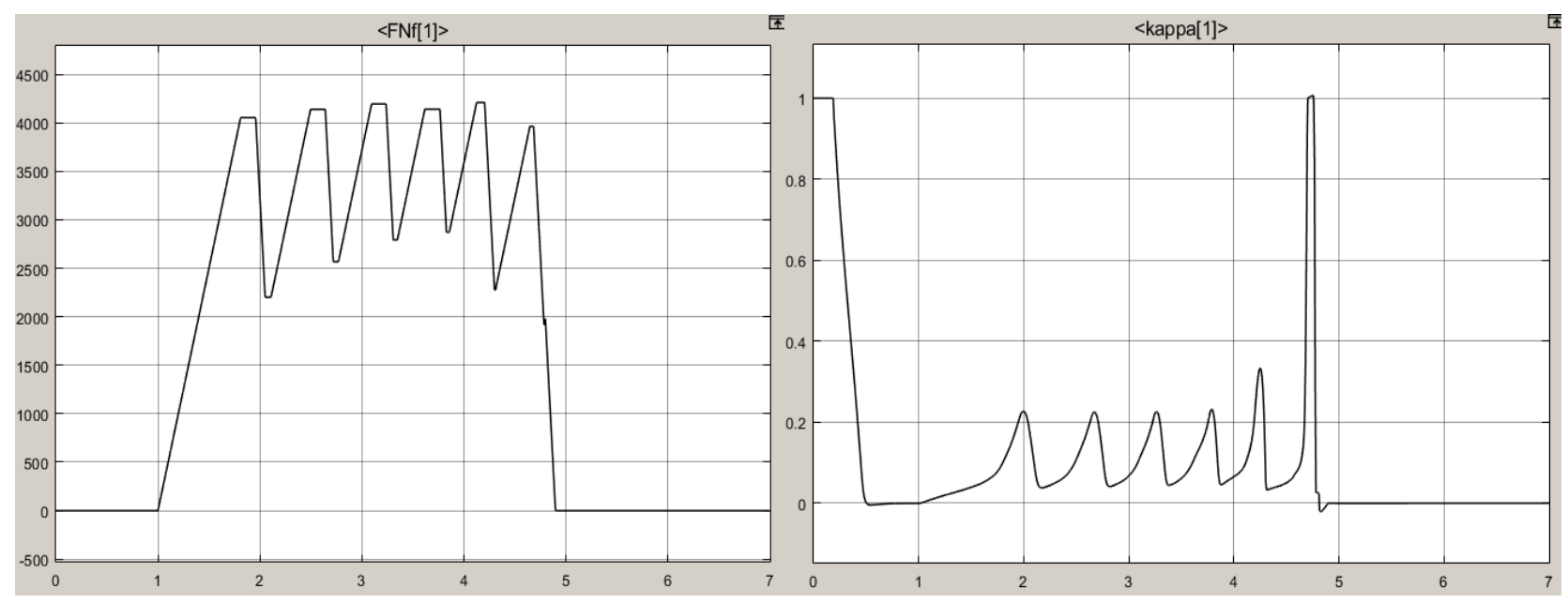

Figure 9. Front-left brake normal force and the corresponding longitudinal slip with 0.01s delayed ABS control

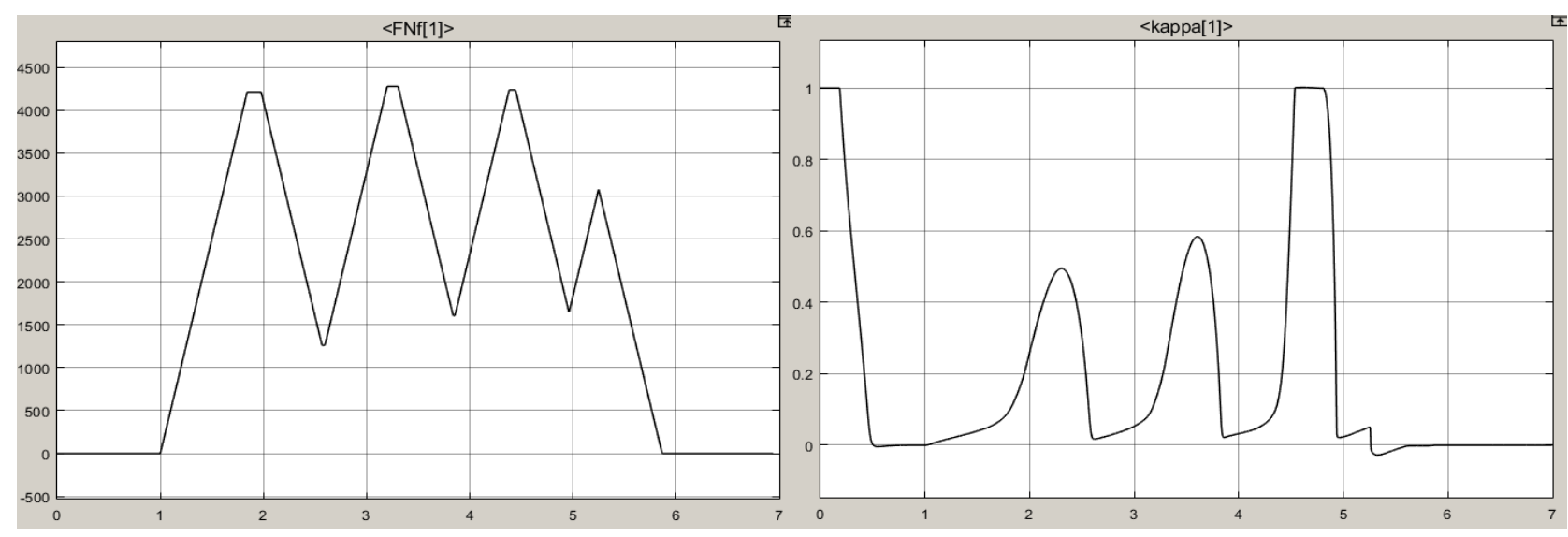

Figure 10. Front-left brake normal force and the corresponding longitudinal slip with 0.03 s delayed ABS control

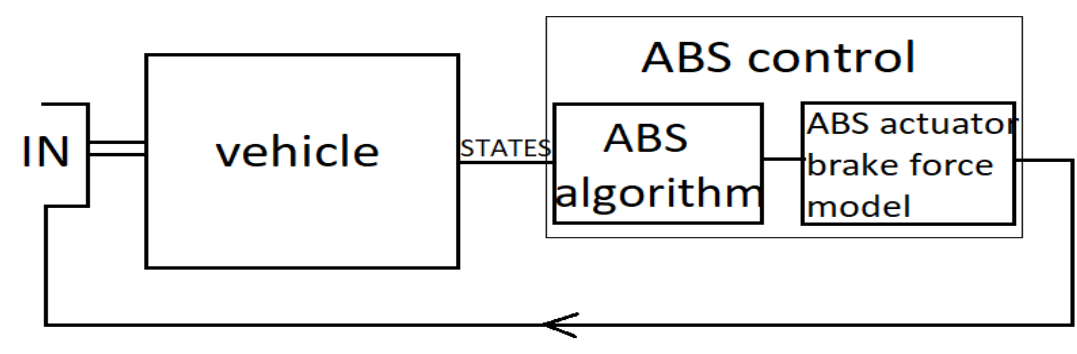

Figure 11. The place of the ABS control model

\section{Acknowledgements}

The work/publication is supported by the EFOP-3.6.1-16-2016-00022 project. The project is cofinanced by the European Union and the European Social. 


\section{References}

[1] M. Carpinelli, Mariano (2016) Automated independent coordinates' switching for the solution of stiff DAEs with the linearly implicit Euler method.Multibody System Dynamics. 36 (1) pp. 67-85.

[2] https://grabcad.com/library/car-chassis-10

[3] G. Rill (2013) TMeasy -- A Handling Tire Model based on a three-dimensional slip approach.

[4] J. Zierath - C. Woernle (2013) Contact modelling in multibody systems by means of a boundary element co-simulation and a Dirichlet-to-Neumann Algorithm. Multibody Dynamics. Springer, Dordrecht. pp. 25-52.

[5] https://www.mathworks.com/matlabcentral/fileexchange/47417-simscape-multibody-contactforceslibrary?

[6] H. Pacejka (2005) Tire and vehicle dynamics. Elsevier.

[7] J. C. M. Fraticelli (2012) Simulink Code Generation: Tutorial for generating C code from Simulink Models using Simulink Coder. Nasa Marshall Space Flight Center.

[8] R. Szabolcsi - J. Menyhárt (2015) Loads Affecting UGVS' Technical Status. Review of the Air Force Academy. 315.

[9] R. Szabolcsi (2016) A New Emergency Landing Concept for Unmanned Aerial Vehicles. Review of the Air Force Academy. 25.

[10] J. Menyhárt - R. Szabolcsi (2016) Support Vector Machine and Fuzzy Logic. Acta Polytechnica Hungarica. 13 (5) pp. 205-220.

[11] L. Pokorádi - J. Menyhárt (2016) Electric vehicles' battery parameter tolerances analysis by fuzzy logic. Applied Computational Intelligence and Informatics (SACI), 2016 IEEE 11th International Symposium on. IEEE.

[12] G. Á. Szíki (2011) Computer program for the calculation of the performance parameters of electromobiles. International Review of Applied Sciences and Engineering. 2 (2) pp. 123-128.

[13] G. Á. Szíki - Gy. Juhász - R. Nagy-Kondor - B. Juhász (2017) Determination and Solution of the Motion of Equation of a Pneumatic Drive Vehicle. Proceedings of the 1st Agria Conference on Innovative Pneumatic Vehicles - ACIPV 2017. pp. 55-58.

[14] G. Á. Szíki - K. Sarvajcz - A Szántó (2017) Dynamic Simulation of a Series Wound DC Motor Applying the Control Design and Simulation Module of Labview. Proceedings of the 5th International Scientific Conference on Advances in Mechanical Engineering - ISCAME 2017. pp. 540-543. 